\title{
La contribución de Enrique Lens Viera al desarrollo musical de la ciudad del apóstol (1854-1945)*
}

\section{The contribution of Enrique Lens Viera to the musical development of the city of apóstol (1854-1945)}

\section{Leslie Freitas de Torres}

Investigadora independiente y miembro del grupo de investigación Organistrum de la Universidad de Santiago de Compostela freitasdetorres@gmail.com ORCID iD: https://orcid.org/0000-0002-4390-0151

\section{RESUMEN}

Enrique Lens Viera (A Coruña/España-1854/Lincoln/Argentina-1945) fue un músico prolijo. Su amplia visión de la realidad de la España decimonónica, en especial de Galicia, permitió que sus esfuerzos en la música estuvieran centrados en distintas parcelas de dicho arte: docente, director, compositor y pianista. Pero, debido a un percance, en 1908 nuestro músico se trasladó a Argentina y jamás regresó a España. Esta investigación, abordada desde la perspectiva de la musicología histórica y del método biográfico narrativo, está basada en las fuentes primarias y secundarias. Su objetivo es proporcionar una aproximación biográfica a la figura de este personaje, que tanto contribuyó al desarrollo de la música gallega decimonónica.

Palabras clave: Enrique Lens Viera, Músico, Santiago de Compostela (España), Lincoln (Argentina), siglo XIX.

\footnotetext{
* Este trabajo es resultado de la investigación realizada en la Universidad de Salamanca (España) con el apoyo del grupo de investigación Organistrum. Fondos documentales de música en los archivos civiles de Galicia (18751951): ciudades del Eje Atlántico (HAR2015-64024-R), proyecto de I+D+i financiado por el MINECO, mediante una ayuda con fondos FEDER de la Unión Europea. Además de haber sido presentado en el $I$ Encuentro de Jóvenes Musicólogos de Salamanca (2-3 de mayo de 2018).
} 


\title{
LESLIE FREITAS DE TORRES
}

\begin{abstract}
Enrique Lens Viera (A Coruña/Spain-1854/Lincoln/Argentina-1945) was a verbose musician. His broad vision of the reality of nineteenth-century Spain, especially Galicia, allowed his efforts in music to be focused on different plots of this art: teacher, director, composer, and pianist. But, due to a mishap, in 1908 our musician moved to Argentina and never returned to Spain. This research, approached from the perspective of historical musicology and the narrative biographical method, is based on primary and secondary sources. Its objective is to provide a biographical approach to the figure of this character, who contributed so much to the development of nineteenth-century galician music.
\end{abstract}

Key Words: Enrique Lens Viera, Musician, Santiago de Compostela (Spain), Lincoln (Argentina), 19th century.

Freitas de Torres, L. (2020). La contribución de Enrique Lens Viera al desarrollo musical de la Ciudad del apòstol (1854-1945). Cuadernos de Investigación Musical, 10, pp. 71-87.

\section{MARCO HISTÓRICO DE LA CIUDAD DEL APÓSTOL}

Durante el siglo XIX, Santiago de Compostela, así como toda España, sufrió una intensa inestabilidad (Villares, 2003, p. 477; Sánchez, 2008, p. 37) por los varios cambios en la estructura política, social y cultural (Martínez, Martínez, 2008, p. 23). Las políticas estuvieron centradas en sus tendencias absolutistas y carlistas (Xunta de Galicia, 2009, p. 16), las cuales estaban "profundamente arraigadas y vinculadas con el clero y la Catedral" (Cancela, 2013, p. 18) ${ }^{1}$. En lo referente a la social, los santiagueses enfrentaron una fuerte crisis alimenticia debido a problemas meteorológicos. Por ser "una comunidad eminentemente agraria caracterizada por una escasa industria" (Moure, 2011, p. 173; Costa, 1983, p. 190; Freire, 2007, p. 2), este trance resultó en un perceptivo retraso económico (Pose, 1992, p. 89), conllevando al fomento del éxodo hacia América².

$\mathrm{Y}$, por último, la cultural, que durante la centuria decimonónica fue un agente activo en el escenario de la reivindicación de la tradición gallega (Amoedo, 2012, pp. 302-303; Carreira, 1999, p. 75), dejando a un lado el monopolio eclesiástico y expandiéndose en varios tipos de manifestaciones (Alén, 2007, p. 51), especialmente musicales: certámenes y orfeones (Carreira, 2008, p. 134); coros (Cortizo, 2001, p. 14); agrupaciones (Pearce, 2015-2016, pp. 56-58); sociedades de esparcimiento (García, 2008, pp. 235-260); cafés (García, 2008, pp. 262-274); cancioneros (Groba, 1974, pp. 11-12); e instituciones, que impartían la enseñanza

\footnotetext{
1 A finales del siglo XIX e inicios del XX, la iglesia proporcionaba a la sociedad gallega el integrismo y el conservadurismo moderado, siendo estos los fertilizantes intelectuales del catolicismo gallego (Carballo, 1995, p. 150).

${ }^{2}$ En 1880 se contabilizaban más de 500 compostelanos embarcados a ultramar (Villa, 2004-2005, p. 17).
} 
musical: Colegio de Sordomudos y de Ciegos (Freitas, 2017), Real Sociedad Económica de Amigos del País (Freitas, 2016a) y Escuela Normal (Alén, 2007, p. 61).

Toda esta efervescencia desembocó en una democratización de la música, dejando de ser "privilegio de las clases pudientes". Por consiguiente, se desarrolló una nueva generación de artistas y se constató la ampliación de las vertientes musicales presentes en el contexto compostelano (Arija, 2011, pp. 36-37), es decir, docencia, dirección, composición e interpretación. Así pues, Enrique Lens Viera se insertó en este escenario, donde sus dotes fueron patentes en estos diferentes ámbitos de la vida musical.

\section{ACERCAMIENTO A LA TRAYECTORIA PERSONAL DE ENRIQUE LENS ViERA}

Nuestro músico nació el 17 de noviembre de 1854 en La Coruña (Baliñas, 1993, pp. 44-51). Inició sus estudios musicales en su ciudad natal, y con apenas ocho años ya daba conciertos de piano (Cañada, 1974). Sobre su formación académica, en 1871 se trasladó a Santiago de Compostela para estudiar en la Universidad Literaria ${ }^{3}$. En esta localidad compaginó su aprendizaje escolar con el arte musical, pues en 1877 ya actuaba en los salones del Casino con destacados músicos de la urbe, como José Courtier y Rafael Tafall ${ }^{4}$. En la década de los setenta, ingresó en la carrera de Farmacia ${ }^{5}$, donde estuvo hasta el curso de $1888-1889^{6}$.

Paralelamente a sus estudios farmacéuticos, contrajo matrimonio con la gallega Carmen Pita Villarnovo que, al concebir a su hijo, Marcial Lens Pita, falleció. Por ello, a los pocos meses de viudo se casó con la señorita Ana Cuena Bermúdez y se trasladó a Huelva, tierra de su esposa, donde obró en la Sociedad Colombina Onubense, en el Casino Minero y en el Círculo Mercantil. Y en 1886, regresó definitivamente a Santiago de Compostela ${ }^{7}$.

\footnotetext{
3 A.H.U.S. Expedientes Personales, Leg. 685, Exp. 3.

${ }^{4}$ Gaceta de Galicia. Diario de Santiago, 17-XII-1877, pp. 2-3.

${ }^{5}$ Marcial Lens Pita cursó la carrera de farmacia en la Universidad de Santiago de Compostela de 1897 a 1900. A.H.U.S. Expedientes Personales, Leg. 684, Exp. 15.

${ }^{6}$ Desafortunadamente, no encontramos su título de farmacéutico en los expedientes conservados en el Archivo Histórico Universitario de Santiago. La última información que se guarda es que Lens solicitó la realización de la asignatura de ejercicios prácticos de clasificación de plantas medicinales para poder aspirar al grado de licenciado en Farmacia, y a los pocos días los realizó, alcanzando un aprobado. Según el noticiero Gaceta de Galicia, Lens «ha recibido el grado de licenciado en Farmacia». Gaceta de Galicia. Diario de Santiago, 16-XI-1889, p. 2; Santiago, 31 de octubre de 1889. A.H.U.S. Expedientes Personales, Leg. 685, Exp. 3.

${ }^{7}$ Gaceta de Galicia. Diario de Santiago, 28-X-1886, p. 2.
} 


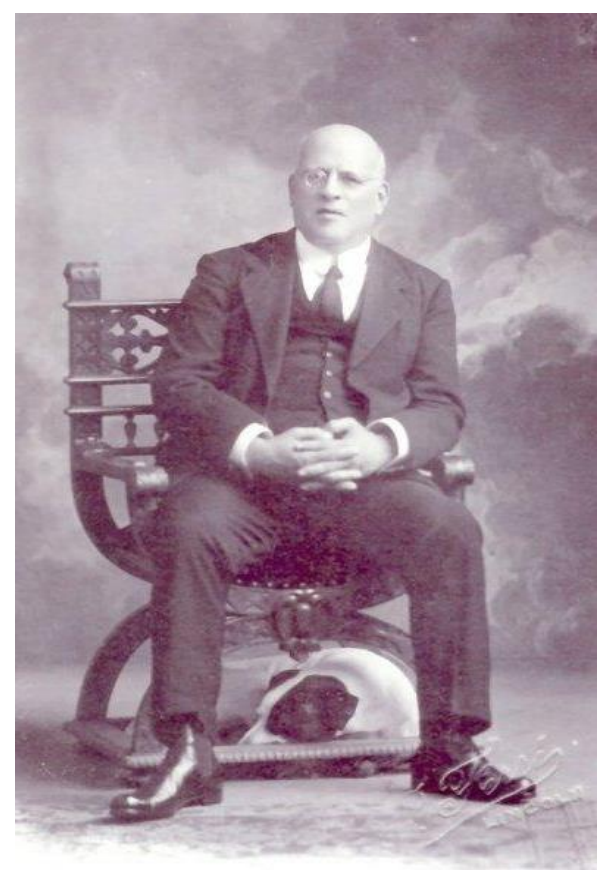

Fig. 1: Foto de Enrique Lens Viera. Archivo Personal de Arturo Lens Closas.

Su labor institucional en la ciudad del Apóstol empezó con la docencia del piano en la escuela de música de la Sociedad Económica, donde posteriormente también ocupó el puesto de director. El músico compartió sus conocimientos con el alumnado en el Colegio de Sordomudos y de Ciegos y en la Escuela Normal. Todas estas actividades docentes, de apoyo al desarrollo humano e intelectual de la población compostelana, le conllevaron a la premiación de la cruz de Caballero de la Real Orden de Isabel la Católica ${ }^{8}$. Asimismo, fueron sus facetas artístico-musicales las que le consagraron como instrumentista, director y compositor.

Su vertiente de ejecutante fue reconocida en Galicia y en las provincias andaluzas, donde gozó de la fama de reputado y notable pianista ${ }^{9}$, siendo invitado a conformar el jurado en varios certámenes ${ }^{10}$. Como director de agrupaciones, realizó un significativo trabajo frente a las distintas formaciones que organizó y/o que fue convidado a dirigir, con especial mención a la segunda Sociedad de Conciertos, creada en Santiago en 1901 por él y por su compañero de profesión, José Gómez Veiga «Curros» (Freitas, 2018a). Y, por último, su faceta compositiva, la cual fue una de las más relevantes en el ámbito regional, poniéndole en el mismo nivel de renombrados músicos gallegos, como Marcial de Adalid (López, Martínez, 2013, p. 215).

Otro aspecto que hay que destacar de este visionario fue su activismo regional (González, 2004, p. 36; Dupláa, 2012, p. 418), pues Enrique Lens mantuvo una estrecha relación de amistad con Alfredo Brañas (Canal, 2006, p. 216), el cual era uno de los ideólogos

\footnotetext{
${ }^{8}$ Blanco y Negro, 17-III-1900, p. 19.

${ }^{9}$ Gaceta de Galicia. Diario de Santiago, 28-X-1886, p. 2.

${ }^{10}$ Gaceta de Galicia. Diario de Santiago, 18-VIII-1894, p. 2. 
de dicho movimiento ${ }^{11}$. Además, sus buenas relaciones políticas le posibilitaron desempeñar funciones oficiales, como el censo ${ }^{12}$ y la fiscalía en la elección municipal ${ }^{13}$. Estas ocupaciones le proporcionaron la medalla de Alfonso XIII ${ }^{14}$. En los últimos años de su residencia en España, ocupó el cargo de corresponsal del periódico Faro de Vigo en Santiago ${ }^{15}$. En 1908, se trasladó a Argentina y fue a vivir en la ciudad de Lincoln, donde estuvo hasta su fallecimiento, 1945.

\section{Su VinCUlaCión CON INSTITUCiONES DOCENTES EN SANTIAGo DE COMPOSTELA}

En la segunda mitad del siglo XIX, entre el reinado de Isabel II (1833-1868) y el de Alfonso XII (1874-1885), surgieron varias instituciones que fueron fundamentales para la evolución y desarrollo de la música española (Nagore, 2013, p. 116) y, por ende, gallega. En Santiago de Compostela se creó la enseñanza musical en algunos establecimientos claves de esta comarca: Real Sociedad Económica de Amigos del País, Colegio de Sordomudos y de Ciegos y Escuela Normal Superior. En las tres instituciones encontramos un punto en común, dicha educación impartida por Enrique Lens Viera.

3.1. Su ETAPA COMO PROFESOR Y DiRECTOR DE LA ESCUELA DE MÚSICA DE LA REAL Sociedad EConómica de Amigos del País de Santiago de Compostela (1886-1908)

Las Sociedades Económicas de Amigos del País fueron una "de las creaciones [...] mejor conocidas del reformismo ilustrado español, [...] creadas en el último tercio del siglo XVIII" (Arias, 2012, pp. 219-220) en varias ciudades del país, donde su prioridad era cambiar el paradigma de la sociedad a través de la educación. En 1784 se instauró una institución de esta naturaleza en Santiago de Compostela (Fernández, 1981, p. 11) y, como forma de complementar la instrucción, en 1877 se creó su escuela de música. De entre sus varios profesores, uno de los más significativos fue Enrique Lens, quien se incorporó a finales de $1886^{16}$.

Con el fin de llevar a cabo una educación informal y "complementaria de la tarea escolar», en el último cuarto de la centuria decimonónica se produjo la aparición de las colonias escolares" (Celada, 2002, p. 107). En Galicia, la primera se realizó en 1893 organizada por la Real Sociedad y la Escuela Normal, ofreciendo a los niños pertenecientes a familias pobres de la región, durante el verano, diversas clases impartidas en el Colegio de

\footnotetext{
${ }^{11}$ El Regional. Diario de Lugo, 16-III-1890, p 2.

12 Gaceta de Galicia. Diario de Santiago, 9-V-1893, p. 3; «Lista de los interventores nombrados ayer por la Junta del censo. Primer Distrito-Primera Sección-Interventores: señores don Ángel Pérez Remesar, Francisco León, Enrique Lens Viera, Luis Gigirey Morentio, Pedro de la Maza Agar, Jesús Diéguez Sanmamed». Gaceta de Galicia. Diario de Santiago, 7-V-1895, p. 2.

${ }^{13}$ Gaceta de Galicia. Diario de Santiago, 14-XI-1893, p. 2.

${ }^{14}$ Gaceta de Galicia. Diario de Santiago, 11-XII-1903, p. 3.

15 Gaceta de Galicia. Diario de Santiago, 25-IV-1905, p. 3.

16 ARSEAPS, Libro de actas, sesión de 30 de diciembre de 1886; Gaceta de Galicia. Diario de Santiago, 8-XII-1886, p. 2; ARSEAPS, Libro de actas, sesión de 29 de diciembre de 1886.
} 


\section{LESLIE FREITAS DE TORRES}

Santiago Apóstol (Pereira, 1983, pp. 199-208). La enseñanza musical de los 32 jóvenes -entre 10 y 13 años- estuvo a cargo de Enrique Lens Viera que, según la prensa, hizo un buen trabajo ${ }^{17}$. En este mismo año, nuestro músico ejerció el papel de director de la escuela de música, a causa de una enfermedad del dirigente ${ }^{18}$. Pero, desgraciadamente, su salud empeoró y falleció ${ }^{19}$. Por ello, la Económica le concedió permanentemente dicho puesto ${ }^{20}$.

Los primeros años de Lens en la coordinación de este Colegio estuvieron marcados por su dirección al frente de la charanga infantil, compuesta por los 32 niños pobres de las escuelas sostenidas por el municipio ${ }^{21}$ y que habían participado de la primera colonia escolar $^{22}$. Los pequeños, que estaban apadrinados por la Económica, tenían clases con los profesores de la escuela de música de la misma, así como con músicos de la Banda Municipal ${ }^{23}$.

Durante el año de 1895, además de la dirección de la banda y de la escuela de música, también continuó ejerciendo la enseñanza de armonía, canto, piano e instrumentos de metal $^{24}$. Asimismo, por su predisposición para la realización de todas estas actividades, la directiva le tributó elogios por el acierto y laboriosidad con que dirigía la academia de la Sociedad ${ }^{25}$.

A finales de $1897^{26}$, nuestro personaje presentó un proyecto de reforma "relativo al reglamento de la escuela de música" ${ }^{27}$. Debido a su estudio, puesto que introducía nuevas asignaturas, así como otros horarios, se suspendieron las clases ${ }^{28}$. Si bien que, al siguiente año la directiva de la Sociedad acordó introducirlo y, por ende, se retomaron las actividades en las aulas de música.

En la distribución de los premios a los alumnos destacados del curso 1904-1905, varios directores de las instituciones gallegas comparecieron para prestigiar dicho acto, y elogiaron el buen trabajo llevado a cabo por Lens en la dirección de la enseñanza musical ${ }^{29}$. Estos encomios llegaron a los oídos de la directiva de la Sociedad Económica, la cual durante su última sesión enalteció a nuestro músico por el estado "altamente satisfactorio de la escuela de música" ${ }^{30}$. Enrique Lens Viera trabajó en esta institución hasta 1908, año en que se marchó a Argentina.

\footnotetext{
17 Gaceta de Galicia. Diario de Santiago, 22-VIII-1893, p. 2.

${ }_{18}$ Gaceta de Galicia. Diario de Santiago, 14-VI-1893, p. 2.

${ }_{19}$ Gaceta de Galicia. Diario de Santiago, 28-XII-1891, p. 2; Gaceta de Galicia. Diario de Santiago, 30-XII-1892, p. 2.

20 ARSEAPS, Libro de actas, sesión de 28 de enero de 1893; Archivo de la Real Sociedad Económica de Amigos del País de Santiago (ARSEAPS), caja 74, 1893, sig. 598; «El señor Lens fue nombrado interinamente [director] en la escuela de [música] de la Sociedad Económica». Gaceta de Galicia. Diario de Santiago, 10-I-1893, p. 3; Archivo de la Real Sociedad Económica de Amigos del País de Santiago (ARSEAPS), caja 74, 1893, sig. 598.

${ }^{21}$ Archivo de la Real Sociedad Económica de Amigos del País de Santiago (ARSEAPS), caja 74, 1894, sig. 610.

${ }^{22}$ Gaceta de Galicia. Diario de Santiago, 15-VI-1894, p. 2; Gaceta de Galicia. Diario de Santiago, 1-VIII-1894, p. 1.

${ }_{23}$ Gaceta de Galicia. Diario de Santiago, 21-VI-1894, p. 3; Gaceta de Galicia. Diario de Santiago, 05-VII-1894, p. 2.

${ }^{24}$ Gaceta de Galicia. Diario de Santiago, 23-V-1896, p. 1.

${ }^{25}$ Gaceta de Galicia. Diario de Santiago, 22-VII-1896, p. 2.

${ }^{26}$ Esta agrupación, que estuvo conocida como petits enfants, durante este año amenizó unas kermeses y participó también de la inauguración del nuevo edificio escolar. El Eco de Santiago, 18-VII-1897, p. 3.

27 ARSEAPS, Libro de actas, sesión de 29 de septiembre de 1897.

28 ARSEAPS, Libro de actas, sesión de 31 de octubre de 1897.

${ }^{29}$ Gaceta de Galicia. Diario de Santiago, 27-VII-1905, p. 2.

${ }^{30}$ Gaceta de Galicia. Diario de Santiago, 11-XII-1905, p. 2.
} 


\subsection{Colegio de Sordomudos y de Ciegos (1890-1908)}

La Ley de Instrucción Pública del 9 de septiembre de 1857 fijó que el gobierno debería proveer la educación para los sordomudos y ciegos en instituciones especiales y, que se implementaría como mínimo una escuela en cada Distrito Universitario de España ${ }^{31}$. Por ende, en 1864 se fundó en Galicia una institución de esta naturaleza, más específicamente en la ciudad del Apóstol, denominada Colegio de Sordomudos y de Ciegos de Santiago de Compostela ${ }^{32}$.

La educación estaba dividida en tres etapas: elemental, de ampliación e industrial (Freitas, 2016b, p. 50). En las dos primeras, los jóvenes con capacidades limitadas asistían a clases de aritmética, religión, historia sagrada, geografía, geometría y música. Siendo esta última centrada en la profesionalización de los deficientes sensoriales, es decir, invidentes. Allí tenían lecciones de solfeo, canto, instrumentos de cuerda, viento, piano y órgano (Freitas, 2017, pp. 17-18).

La instrucción de la música estuvo a cargo de varios profesores a lo largo de su historia, siendo su plantilla compuesta por dos docentes-un principal y un segundo o ayudante-y un copista: Miguel García (1868-1870), Juan Lorenzo González (1868-1873), Joaquín María Castaños (1869-1870), Gregorio Barcia (1870-1890), Gerardo Gómez Veiga (enero-junio 1890) (Freitas, 2018b, pp. 163-165) y Ramón Bugueiro (1870-1890). Por fallecimiento de este último, se produjo la contratación de nuestro músico como maestro principal ${ }^{33}$.

Como de costumbre, anualmente se realizaba inspecciones de las dependencias del Colegio y de las enseñanzas impartidas, inspección donde el alumnado demostraba al encargado de la visita su desarrollo en las diferentes disciplinas. En la de 1891, este papel fue desempeñado por el rector de la Universidad que por tres días estuvo examinándole. En lo concerniente a las clases de música, el profesor principal (Enrique Lens) y su ayudante (José Gómez Veiga) fueron los responsables de presentar los adelantos de los estudiantes. Al final, “el sr. rector felicitó al sr. Lens, y [...] demostró su complacencia por el brillante estado de todos los alumnos" 34 .

Tras este reconocimiento, en 1904 dichos profesores formaron con los alumnos un terceto y un sexteto con el afán de apoyar la velada realizada por la sociedad coral Unión Artística y, según la prensa, todos quedaron asombrados con las obras interpretadas ${ }^{35}$. Estos logros sirvieron de motivación para que los docentes los llevasen a participar en el Certamen de Rondallas de La Coruña, donde ganaron el primer premio ${ }^{36}$. Sin justificación previa, en julio de 1908 el profesor Enrique Lens Viera abandonó su plaza ausentándose de la localidad,

\footnotetext{
31 Artículo $6^{\circ}$ y $108^{\circ}$. F.U., S.H. 583. Memoria sobre el Estado actual del Colegio de Sordo-mudos y de ciegos de Santiago, 1867.

${ }^{32}$ F.U., S.H. 583. Memoria sobre el Estado actual del Colegio de Sordo-mudos y de ciegos de Santiago, 1867.

${ }^{33}$ Santiago, 14 de junio de 1890. F.U., S.H. 6869. Escuelas especiales Sordomudos/Correspondencia General.

${ }^{34}$ Gaceta de Galicia. Diario de Santiago, 6-VIII-1891, p. 2.

35 Gaceta de Galicia. Diario de Santiago, 2-II-1904, p. 5.

${ }^{36}$ Gaceta de Galicia. Diario de Santiago, 21-VII-1912, p. 2; Archivo del Colegio de Educación Especial Manuel López Navalón, caja 3.
} 


\section{LESLIE FREITAS DE TORRES}

“según es público para no volver" ${ }^{\gg 7}$. Con eso, la labor de nuestro músico se terminó en esta institución.

\subsection{Escuela Normal de Santiago de Compostela (1894-1908)}

"Por la reglamentación del Real Decreto de 30 de marzo de 1849", se impuso el establecimiento de Escuelas Normales Superiores en las capitales de Distritos Universitarios españoles $^{38}$. Por consiguiente, el director de la Universidad santiaguesa, Juan José Viñas, implementó una institución de esta naturaleza en la ciudad del Apóstol, en el antiguo colegio San Xerome (Fariña, 1996, p. 40). La misma fue creada para los jóvenes, de más de 15 años, que deseaban alcanzar una formación superior en el ámbito de la docencia (Porto, 2009, p. 338).

Las clases de música en la Escuela Normal Superior de Maestros de Santiago dio inicio en 1878 (Alén, 2007, p. 61), si bien que nuestro músico ingresó como docente en $1894^{39}$, posterior a la renuncia del anterior profesor Manuel Chaves. Sin embargo, una duda sobre la incompatibilidad de su docencia en dos instituciones oficiales de enseñanza, es decir, Escuela Normal y Colegio de Sordomudos y de Ciegos, inquietó a la dirección y esta inmediatamente consultó al rectorado de la Universidad a fin de aclararla. En respuesta, se comunicó que como en la Normal Lens recibiría una retribución en el concepto de gratificación y no un sueldo fijo, ambos trabajos eran compatibles ${ }^{40}$.

Tras esta contestación, nuestro músico pudo empezar su labor. No obstante, en 1899 con el ideal de ampliar las ofertas de enseñanzas especiales, tal como dictar la forma de ingreso de los profesores, es decir, comprobando sus méritos ya que la prensa continuamente la cuestionaba, la dirección realizó oposiciones para las plazas de francés, dibujo y caligrafía (juntos) y música ${ }^{41}$. Lo que se refiere a esta última, Lens Viera no consiguió conservar su cargo y tuvo que presentarse como uno de los opositores ${ }^{42}$. Y la resolución le fue favorable, confirmando su competencia docente frente a los demás aspirantes ${ }^{43}$. Así que Lens Viera obró en dicha institución hasta 1908, año que abandonó la docencia en la Escuela Normal para trasladarse a Argentina ${ }^{44}$.

\footnotetext{
${ }^{37}$ Santiago, 2 de julio de 1908. F.U., S.H. 564. Escuelas Especiales Sordomudos/Correspondencia General. 38 http://www.usc.es/es/centros/cc educacion/historia.html. Consultado 30/01/2018].

39 «D. Enrique Lens, nombrado por orden de 8 del mes corriente profesor de música en la Escuela Normal de esta ciudad con la retribución anual de 750 pesetas». A.H.U.S. F.U. 3235. Correspondencias; Gaceta de Galicia. Diario de Santiago, 18-III-1894, p. 2.

40 A.H.U.S. F.U. 3235. Correspondencias.

${ }^{41}$ Santiago, 10 de mayo de 1899. A.H.U.S. F.U. 3236. Correspondencias; Gaceta de Galicia. Diario de Santiago, 3VIII-1899, p. 2.

${ }^{42}$ Santiago, 10 de agosto de 1899. A.H.U.S. F.U. 3236. Correspondencia.

43 A.H.U.S. F.U. 3236. Correspondencia.

${ }^{44}$ Gaceta de Galicia. Diario de Santiago, 23-IX-1908, p. 3; A.H.U.S. F.U. 3236. Correspondencia.
} 


\section{INTÉRPRETE Y DIRECTOR DE FORMACIONES MUSICALES}

Una de las principales novedades del siglo XIX “fue la progresiva segmentación de los repertorios y los gustos, que acabaron prefigurando distintos tipos de conciertos en función del público al que se destinaban: concierto de música de cámara, sinfónico, coral, de virtuoso, café-concierto y la gala formada por extractos de ópera y canciones” (Marín, 2013, p. 90). Y para ello, se constituyeron diferentes agrupaciones instrumentales, destacándose en la geografía gallega desde los solos hasta las grandes formaciones -orquestas o sociedades de conciertos- (Villa, 2004-2005, p. 30), las cuales contaron con la activa participación de nuestro músico tanto interpretando como dirigiendo.

Las presentaciones que Lens realizó, como músico y/o director, se llevaron a cabo en distintos lugares: Teatro Principal, centros de esparcimiento (Casino, Sociedad Recreo Artístico e Industrial, Círculo Mercantil y Ateneo León XIII) y, en menor número, en el Seminario al igual que en el balneario de Cuntis. Su primera actuación en Santiago de Compostela, posterior a su regreso de Huelva, fue una velada literario-musical en el Teatro Principal llevada a cabo a su propio beneficio, donde Lens realizó algunos solos al piano con obras de compositores del romanticismo europeo, como: Weber y Gottschalk ${ }^{45}$.

En los centros de esparcimiento su contribución era siempre requisitada, como: en el Casino, donde fue contratado como pianista oficial ${ }^{46}$; en la Sociedad Recreo Artístico e Industrial, en que organizaba veladas musicales con sus discípulos y con agrupaciones de renombre en la ciudad (sexteto Courtier y la orquesta Curros $^{47}$ ); en el Círculo Mercantil que, además de dar a conocer por primera vez una de sus obras (Fantasía Impromptu ${ }^{48}$ ), también contribuyó en las celebraciones religiosas ${ }^{49}$; o en el Ateneo León XIII, donde amenizaba sus conferencias $^{50}$.

Sus actuaciones en instituciones eclesiásticas son muy escasas, ya que el piano no se contemplaba en la iglesia (Ortega, 2013, p. 130). Por ello, la única en un establecimiento de esta naturaleza -Seminario Diocesano- fue en una sesión literaria-religiosa en honor de la Virgen Santísima de los Dolores, donde Lens amenizó el acto ${ }^{51}$. Ya en el Balneario de Cuntis, como nuestro músico anualmente veraneaba allí, este apoyaba algunas veladas que se llevaban a cabo en el salón de la Virgen ${ }^{52}$.

Asimismo, no podemos dejar de citar su trabajo como director, juntamente con su compañero de profesión José Gómez Veiga «Curros», al frente de la Sociedad de Conciertos de Santiago de Compostela -1901-, donde ambos eran los responsables de la organización, producción, programación y dirección de los conciertos, cuyo propósito era ofrecer a la

\footnotetext{
45 Gaceta de Galicia. Diario de Santiago, 14-XI-1886, p. 3.

46 Gaceta de Galicia. Diario de Santiago, 3-XII-1886, p. 2.

${ }^{47}$ Gaceta de Galicia. Diario de Santiago, 6-XII-1890, p. 2; Gaceta de Galicia. Diario de Santiago, 9-XII-1890, p. 2; Gaceta de Galicia. Diario de Santiago, 9-XII-1890, p. 3.

48 El Alcance, 8-I-1898, p. 1.

49 Gaceta de Galicia. Diario de Santiago, 6-I-1900, p. 1.

${ }^{50}$ El Alcance, 12-III-1898, p. 1.

${ }^{51}$ Gaceta de Galicia. Diario de Santiago, 29-III-1887, p. 2; Gaceta de Galicia. Diario de Santiago, 2-IV-1887, p. 2.

${ }^{52}$ Gaceta de Galicia. Diario de Santiago, 21-VIII-1890, p. 2.
} 


\section{LESLIE FREITAS DE TORRES}

comunidad santiaguesa presentaciones periódicas con obras de grandes maestros internacionales, como: Haydn o Beethoven (Freitas, 2018a, p. 291).

\section{BREVE REPASO SOBRE SU FACETA DE COMPOSITOR}

Enrique Lens presenta una producción musical enfocada al folclore gallego (López, Martínez, 2013, pp. 191-205), donde emplea las melodías y ritmos sin transcribirlas literalmente y sí como fuente de inspiración (Amoedo, 2012, p. 313). En sus textos utilizaba el idioma gallego y alteraba la composición de las plegarias, polkas, valses, etc., que eran características de las habituales danzas de la época, y las convertía en obras como la Fantasía Impromptu o el vals de concierto Recuerdos de Lourizán, gracias a una escritura virtuosa que buscaba la explotación de los recursos instrumentales del piano (Carreira, 1990) ${ }^{53}$.

Diferente de la mayoría de los músicos/compositores gallegos, Lens se centró en obras para su instrumento solo o como acompañamiento. Quizás eso ocurrió porque, del mismo modo que el piano era para las familias pudientes uno de los símbolos de estatus de educación y cultura, sus composiciones serían compradas con facilidad, respondiendo así a una demanda social concreta. Eso lo hace singular, ya que no era una práctica recurrente entre sus contemporáneos, a poner algunos ejemplos entre otros posibles, José Gómez Veiga «Curros» (1864-1946) era violinista y compuso dúos (voz y piano/violín y piano), quintetos de cuerdas, sextetos de cuerdas, al igual que música para orquesta y banda (Freitas, 2018a, pp. 315-335); Juan Montes Capón (1840-1899) era pianista y organista y escribió para piano, banda, cuarteto de cuerdas y orfeón (Villanueva, Trillo, 2016); o Andrés Gaos (1874-1959) era violinista y compuso dúos (violín y piano/violoncello y piano), así como obras escénicas, sinfónico-corales, para orquesta de cuerdas, piano solo, entre otras (Painceira, 2013, p. 8).

Sin una exhaustiva labor, pues nuestro objetivo no es proporcionar un catálogo musical, realizamos una recopilación de sus obras. Su mayor legado hoy se halla disperso en las distintas bibliotecas gallegas y de Madrid, lo que es algo inusual para los compositores de esta época, ya que sus descendientes solían depositarlas en un único fondo que, dependiendo de la cantidad de documentos, llevaría su nombre. Las excepciones son las músicas Serenata y Soño era, puesto que la primera se encuentra en el archivo personal del investigador Alejo Amoedo (2012, p. 103) y la segunda en el de los herederos españoles de nuestro músico, la cual fue editada por Xoán Manuel Carreira (1996, pp. 253-260).

\footnotetext{
${ }^{53}$ Biblioteca Municipal de Estudios Locales de A Coruña, sig. RC AR 24.195.
} 
LA CONTRIBUCIÓN DE ENRIQUE LENS VIERA AL DESARROLLO MUSICAL

DE LA CIUDAD DEL APÓSTOL (1854-1945)

\begin{tabular}{|c|c|c|c|c|}
\hline Localización & Título & & Fecha & Instrumentos \\
\hline $\begin{array}{l}\text { Biblioteca Municipal de } \\
\text { Estudos Locais de A Coruña }\end{array}$ & ¿Quéresme? [sic.] & $\begin{array}{l}\text { música } \\
\text { impresa }\end{array}$ & --------------- & voz y piano ${ }^{54}$ \\
\hline $\begin{array}{l}\text { Biblioteca Municipal de } \\
\text { Estudos Locais de A Coruña }\end{array}$ & Rhapsodia gallega & $\begin{array}{l}\text { música } \\
\text { impresa }\end{array}$ & --------------- & piano $^{55}$ \\
\hline $\begin{array}{l}\text { Biblioteca Municipal de } \\
\text { Estudos Locais de A Coruña }\end{array}$ & iA neniña & $\begin{array}{l}\text { música } \\
\text { impresa }\end{array}$ & 1897 & vOz y piano ${ }^{56}$ \\
\hline $\begin{array}{l}\text { Biblioteca Municipal de } \\
\text { Estudos Locais de A Coruña }\end{array}$ & Salayos & $\begin{array}{l}\text { música } \\
\text { impresa }\end{array}$ & $-2-2-1$ & voz y piano ${ }^{57}$ \\
\hline Municipal de Moaña & Malenconia [sic.] & $\begin{array}{l}\text { música } \\
\text { impresa }\end{array}$ & --------------- & voz y piano ${ }^{58}$ \\
\hline Biblioteca Nacional de España & Recuerdos de Lourizán & $\begin{array}{l}\text { música } \\
\text { notada }\end{array}$ & 1892 & piano $^{59}$ \\
\hline Biblioteca Nacional de España & ¡Sarantellos! & $\begin{array}{l}\text { música } \\
\text { notada }\end{array}$ & 1891 & piano $^{60}$ \\
\hline Biblioteca Nacional de España & Alma hermosa & $\begin{array}{l}\text { música } \\
\text { notada }\end{array}$ & 1901 & piano $^{61}$ \\
\hline $\begin{array}{l}\text { Archivo Personal de Alejo } \\
\text { Amoedo }\end{array}$ & Serenata & manuscrito & 1893 & Piano $^{62}$ \\
\hline $\begin{array}{l}\text { Archivo Personal de los } \\
\text { herederos de Enrique Lens } \\
\text { Viera }\end{array}$ & Soño era & $\begin{array}{l}\text { Música } \\
\text { notada }\end{array}$ & -------- & piano $^{63}$ \\
\hline
\end{tabular}

Tabla 1: Partituras de Enrique Lens Viera. Elaboración propia

\footnotetext{
${ }^{54}$ Biblioteca Municipal de Estudos Locais de A Coruña, deposito general, sig. RC MI 5.1.24.

55 Biblioteca Municipal de Estudos Locais de A Coruña, deposito general, sig. RC MI 5.1.19.

56 Biblioteca Municipal de Estudos Locais de A Coruña, deposito general, sig. RC MI 5.1.22; Biblioteca Municipal de Estudos Locais de A Coruña, depósito general, sig. RC MI 5.1.23; Biblioteca Digital Hispánica, sala Barbieri sig. M. MARDONES/37.

${ }^{57}$ Biblioteca Municipal de Estudos Locais de A Coruña, deposito general, sig. RC MI 5.1.20.

58 Biblioteca Municipal de Moaña, sig. C-1 051; Biblioteca Municipal de Estudos Locais de A Coruña, depósito general, sig. RC MI 5.1.26.

${ }_{59}$ Biblioteca Nacional de España, sala Barbieri, sig. MP/2696/20.

${ }^{60}$ Biblioteca Nacional de España, sala Barbieri, sig. MP/315/1.

${ }^{61}$ Biblioteca Nacional de España, sala Barbieri, sig. MMIC RO/5044 (22); Biblioteca Municipal de Estudos Locais de A Coruña, deposito general, sig. RC MI 53.2.6.

62 Amoedo, 2012, p. 103.

${ }^{63}$ Carreira, 1996, pp. 253-260.
} 


\section{LESLIE FREITAS DE TORRES}

\section{6. ¿POR QUÉ LENS EMIGRó A ARgentina?}

Entre los años de 1900-1930 Galicia registró un gran índice migratorio, los destinos comúnmente elegidos eran los países de América del sur: Brasil, Cuba, México, Uruguay, Colombia y Argentina (Vázquez, 1988, pp. 86-87). En esta última cuna, el colectivo de gallegos en el primer tercio del siglo XX estuvo "alrededor de un $50-55 \%$ del contingente de españoles residentes en Buenos Aires” (Núñez, 2007, pp. 28-29) ${ }^{64}$.

Por lo general, las causas que producían este traslado eran consecuencia del desajuste socioeconómico, huidas del servicio militar obligatorio, las propagandas presuntamente ilusorias de una vida mejor y por la creación de cadenas migratorias a través de conocidos y/o parientes (Campos, 1993-1994, pp. 133-134). En los casos de los músicos, la emigración ocurría para consolidar los estudios y buscar su propio estilo (Alén, 2004, p. 46), puesto que "la estancia de formación en el extranjero [...] [contaba] con un peso muy importante, dadas las limitaciones de la educación musical española" (Carreras, 2018, p. 56). Asimismo, no era habitual, pero había quien la utilizaba como válvula de escape para virar página y empezar una nueva vida sin que nadie supiera de su pasado, siendo este el caso de nuestro músico.

El motivo de su traslado fue su expulsión de la escuela de música de la Económica. Lo que sucedió fue que en junio de 1908 Enrique Lens Viera y otros dos maestros de la misma institución, solo que de distintas asignaturas ${ }^{65}$, realizaron un acto digno de reprobación. Y las medidas tomadas fueron la suspensión de uno y la separación definitiva de los otros dos, siendo uno de estos nuestro músico ${ }^{66}$.

Desgraciadamente las actas de dicha institución, tal como los periódicos de la época, no relatan que hecho fue ese, ya que siempre que se refieren al mismo dicen: "como todos tenemos conocimiento". No obstante, sospechamos que fue algún problema con las alumnas de la escuela de música de dicho establecimiento que iban a prestar exámenes, ya que de entre los acuerdos tomados posteriores a su salida se determinó que fuera las "profesoras las encargadas de las enseñanzas de señoritas" ${ }^{\prime 67}$.

\footnotetext{
${ }^{64}$ Gaceta de Galicia. Diario de Santiago, 30-III-1895, p. 2.

65 «En el expediente instruido por la Comisión Curadora de las escuelas de la Sociedad contra el director y profesor de la escuela de música D. Enrique Lens y los mozos Manuel García Lafuente y Andrés Rilo Medan, se acordó por aquella y decretado por esta dirección separar de sus respectivos cargos a los dos primeros dándoles de baja en la nómina del mes actual, e imponer al tercer suspensión [sic.] de un mes de haber mensual [...]. Santiago, 10 de julio de 1908». Archivo de la Real Sociedad Económica de Amigos del País de Santiago (ARSEAPS), caja 88, 1908, sig. 789; «Por razones de todos conocidas, fue separado el director de la escuela de música D. Enrique Lens». Archivo de la Real Sociedad Económica de Amigos del País de Santiago (ARSEAPS), caja 88, 1908, sig. 788.

${ }^{66}$ ARSEAPS, Libro de actas, sesión de 22 de junio de 1908; ARSEAPS, Libro de actas, sesión ordinaria de 29 de septiembre de 1908.

${ }^{67}$ ARSEAPS, Libro de actas, sesión de 22 de junio de 1908; ARSEAPS, Libro de actas, sesión de 31 de agosto de 1908.
} 


\section{CONCLUSIONES}

La investigación llevada a cabo se ha limitado al estudio del transcurso de Enrique Lens Viera en la urbe gallega, para la cual, tras una exhaustiva búsqueda de las fuentes primarias, gran parte de ellas inéditas hasta el presente momento, además de las secundarias, hemos erigido un acercamiento a la biografía de este músico, explicando y contextualizando con hechos de las distintas épocas. Tratando de su trayectoria personal, su labor de profesor de música en las instituciones santiaguesas, su desarrollo como intérprete y director de las formaciones musicales, su faceta de compositor y, por último, el motivo de su traslado a Argentina.

Asimismo, quizás las dos aportaciones más significativas de nuestra investigación se demarcan primero a su vertiente compositiva, en que aportamos una tabla inédita con todas sus obras encontradas hasta el presente momento. Esta contribución permite que se rescate la memoria de este músico, y esperamos que a partir del instante que este escrito salga a luz los investigadores percátense de la importancia de Lens para la historia de la música gallega. Y la segunda, en dar a conocer por primera vez el motivo por el cual Lens se trasladó de España, aclarando así las dudas de varios investigadores que durante años estuvieron cogitando el porqué de su viaje repentino.

Para este escrito nos limitamos a tratar de la figura de Lens en Galicia. Sin embargo, para conocer a este personaje por completo es necesario dibujar su vida en las otras localidades donde vivió, siendo estas Huelva (España) y Lincoln (Argentina), las cuales ya estamos trabajando gracias a la cooperación del grupo de investigación de la Universidad de Santiago de Compostela que hacemos parte, denominado Organistrum: Fondos documentales de música en los archivos civiles de Galicia (1875-1951): ciudades del Eje Atlántico (HAR2015-64024-R), proyecto de I+D+i financiado por el MINECO, mediante una ayuda con fondos FEDER de la Unión Europea.

\section{BibLIOGRAFÍA}

Alén Garabato, M. del P. (2004). Breve história da música galega. Vigo: A Nosa Terra.

Alén Garabato, M. del P. (2007). Reflexiones sobre un siglo de música gallega (ca.18081916). Revista de Musicología, XXX, 1, pp. 49-102.

Amoedo, A. (2012). Dúas obras inéditas para piano só dos compositores Felipe Paz Carbajal e Enrique Lens Viera. En M. Capelán, Costa Vázquez, L., Garbayo Montabes, J., Villanueva, C. (Eds.), Os Soños da Memoria. Documentación Musical en Galicia: Metodoloxías para o estúdio, pp. 301-314. Pontevedra: Diputación de Pontevedra. 


\section{LESLIE FREITAS DE TORRES}

Arias de Saavedra Alías, I. (2012). Las Sociedades Económicas de Amigos del País: Proyecto y Realidad en la España de la Ilustración. Obradoiro de Historia Moderna, 21, pp. 219-245.

Arija Soutullo, M. R. (2011). Reveriano Soutullo Otero. El alma lírica de la música gallega. Vida y Obra. Pontevedra: Diputación de Pontevedra.

Baliñas, M. (1993). A vida musical en Santiago. En X. M. Carreira Antelo, C. Magán (Eds.). Ángel Brage. Memoria musical dun século, pp. 44-51. Santiago de Compostela: Consorcio de Santiago.

Campos Álvarez, J. R. (1993-1994). La emigración gallega a América (1880-1930): integración y retorno. Minius, II-III, pp.133-148.

Canal I Morell, J. (2006). Banderas blancas, boinas rojas: una historia política del carlismo, 1876-1939. Madrid: Marcial Pons Ediciones de Historia.

Cancela Montes, A., Cancela Montes, B. (2013). La saga Courtier en Galicia. Santiago de Compostela: Alvarellos.

Carballo, F. (1995). A Igrexa galega. Vigo: Edicións A Nosa Terra.

Carreira Antelo, X. M. (1974). Lens Viera, Enrique. En S. Cañada Acebal (ed.). Gran Enciclopedia Galega, t. 25, p. 225. Gijón: Silverio Cañada.

Carreira Antelo, X. M. (1990). Enrique Lens Viera. Notas al Programa (folleto).

Carreira Antelo, X. M. (1996). As melodias galegas de Enrique Lens. En M. X. Rodríguez Galdo, X. R. Barreiro Fernández, V. Peña Saavedra (Eds.). Estudios Migratorios, pp. 241266. Santiago de Compostela: Consello da Cultura Galega: Arquivo da Emigración Galega: Universidade de Santiago de Compostela.

Carreira A., X. M. (1999). Las seis baladas de Juan Montes en el contexto internacional de la canción de arte de la Belle Époque. Actas del Congreso sobre Juan Montes, pp. 69-101. Santiago de Compostela: Xunta de Galicia.

Carreira A., X. M. (2008). A música profana en Galicia na restauración. En Congreso Galicia nos tempos do 98, pp. 133-152. Santiago de Compostela: Xunta de Galicia.

Carreras, J. J. (2018). Movilidad, redes, representaciones. En J. J. Carreras (Ed.). Historia de la música en España e Hispano América. La música en España en el siglo XIX, pp. 51-74. Madrid: Fondo de Cultura Económica de España. 


\section{LA CONTRIBUCIÓN DE ENRIQUE LENS VIERA AL DESARROLLO MUSICAL}

DE LA CIUDAD DEL APÓSTOL (1854-1945)

Celada Parandones, P. (2002). Las colonias escolares leonesas (1895, 1896 y 1936). Tierras de Léon: Revista de la Diputación Provincial, XL, 114, pp. 105-142.

Cortizo, M. E., Sobrino Sánchez, R. (2001). Asociacionismo musical en España. Cuadernos de Música Iberoamericana, VIII-IX, pp. 11-16.

Costa Rico, A. (1983). Instituciones para la formación de los maestros gallegos en los finales del siglo XIX. Historia de la Educación, II, pp. 189-198.

Dupláa, C. (2012). Rosalía de Castro y el Rexurdimento Gallego: posibles conexiones con la Renaixenca catalana. Actas do Congreso de Estudios sobre Rosalía de Castro e o seu tempo (III), pp. 413-418. Santiago de Compostela: Consello da Cultura Galega: Universidad de Santiago de Compostela.

Fariña Casaldarnos, M. del C. (1996). O Instituto «Arcebispo Xelmiréz»» de Santiago de Compostela. História Documental 1845-1857. Santiago de Compostela: Servicio de Publicacións da Universidade de Santiago de Compostela.

Fernández Casanova, M. del C. (1981). La Sociedad Económica de Amigos del País de Santiago en el siglo XIX. Un estudio de la organización interna y de su actuación en favor de Galicia. A Coruña: Edicións do Castro.

Freire Esparís, M. del P. (2007). Una aproximación a los procesos de cambio en la Galicia rural a través del análisis de los municipios de Brión y Padrón (1850-1970). Revista Galega de Economía, XVI, pp. 1-25.

Freitas de Torres, L. (2016a). La escuela de música de la Real Sociedad Económica de Amigos del País de Santiago de Compostela (1877-1953). Revista de Musicología Argentina, 17, pp. 131-150.

Freitas de Torres, L. (2016b). El Colegio de Sordomudos y de Ciegos de Santiago de Compostela (18642016). Madrid: L.A.

Freitas de Torres, L. (2017). El sonido de la beneficencia emitido por las clases de música del Colegio de Sordomudos y de Ciegos de Santiago de Compostela (1868-1957). Madrid: L.A.

Freitas de Torres, L. (2018a). José Gómez Veiga «Curros». El rostro de la música compostelana. Santiago de Compostela: Guiverny.

Freitas de Torres, L. (2018b). Una aproximación a la biografía del músico gallego Gerardo Gómez Veiga Curros (1866-1921). Revista Catalana de Musicología, 11, pp. 159-175. 


\section{LESLIE FREITAS DE TORRES}

García Caballero, M. (2008). La vida musical en Santiago a finales del siglo XIX. Santiago de Compostela: Alvarellos.

González Millán, X. (2004). E. Pardo Bazán y su imagen del rexurdimento cultural gallego en la revista de Galicia. La Tribuna: Cuadernos de Estudos da Casa Museo Emilia Pardo Bazán, 2, pp. 35-64.

Groba y Groba, R. (1974). El maestro Soutullo y la música gallega. La Coruña: Real Academia de Bellas Artes de Nuestra Señora del Rosario.

López-Suevos Hernández, B. \& Martínez Martínez, M. R. (2013). Eugenia Osterberger (Mme. Saunier). Una aportación femenina a la creación musical gallega. Nalgures, IX, pp. 151-232.

Marín, M. Á. (2013). Tendencias y desafíos de la programación musical. BROCAR, 37, pp. 87-104.

Martínez, N. I. \& Martínez, I. L. (2008). La música del siglo XIX. Una herramienta para su descripción bibliográfica. Madrid: Biblioteca Nacional.

Moure Pazos, I. (2011). El proyecto de Ricardo Velázquez Bosco para Santiago de Compostela: La Escuela de Sordomudos y Ciegos (1905-1925). Ars Longa, 20, pp. 173182.

Nagore Ferrer, M (2013). Música y músicos navarros en los dos siglos XIX y XX. Revista TKAsnabi, 25, pp. 115-121.

Núñez Seixas, J. M. (2007). Un panorama social de la inmigración gallega en Buenos Aires (1750-1930). En M. V. Ameztoy (Coord.). En Buenos Aires Gallega. Inmigración, pasado y presente, pp. 73-96. Buenos Aires: Comisión para la Preservación del Patrimonio Cultural de la Ciudad Autónoma de Buenos Aires.

Ortega López, Á. (2013). Los órganos de la Catedral de Calahorra. Kalakorikos, 18, pp. 129-145.

Painceira Luaces, V. (2013). La revitalización de la figura de Andrés Gaos en Galicia. (Trabajo de Fin de Máster). Oviedo: Universidad de Oviedo.

Pearce Pérez, M. del C. (2015-2016). La actividad musical en los espacios religiosos de La Habana en la segunda mitad del siglo XIX. (Trabajo Final de Máster). Oviedo: Universidad de Oviedo. 


\section{LA CONTRIBUCIÓN DE ENRIQUE LENS VIERA AL DESARROLLO MUSICAL}

DE LA CIUDAD DEL APÓSTOL (1854-1945)

Pereira Domínguez, M. del C. (1983). La primera colonia escolar de Galicia. Historia de la Educación, II, pp. 199-208.

Porto Ucha, Á. S. (2009). La educación especial en la formación de los maestros y maestras en el primer tercio del siglo XX. Algunos datos referidos a Galicia. En M. Reyes Berruezo \& S. Conejero López (Coord.). El largo camino hacia una educación inclusiva: la educación especial y social del siglo XIX a nuestros días: XV Coloquio de Historia de la Educación, Pamplona-Iruña, 29, 30 de junio y 1 de julio, vol. I, pp. 335-344. Pamplona-Iruña: Universidad Pública de Navarra.

Pose Antelo, J. M. (1992). La economía y la sociedad compostelana a finales del siglo XIX. Santiago de Compostela: Servicións de Publicacións e Intercambio Cientifico da Universidade de Santiago de Compostela.

Sánchez Huedo, O. (2008). La Banda Municipal de Música de Albacete: Desde sus orígenes hasta la primera década del siglo XX. (Tesis Doctoral). Salamanca: Universidad de Salamanca.

Vázquez González, A. (1988). La emigración gallega. Migrantes, transporte y remesas. En N. SÁNCHEZ-ALBORNOZ (comp.). Españoles hacia América. La emigración en masa, 1880-1930, pp. 80-104. Madrid: Alianza América.

Villanueva, C. \& Trillo, J. (2016). Obras para piano. Juan Montes Capón. Pontevedra: Dos Acordes.

Villa Vieiro, F. (2004-2005). La obra musical del canónigo Marcelino Sempere en la Catedral de Santiago de Compostela (1879-1884). (Diploma de Estudios Avanzados). Santiago de Compostela: Universidad de Santiago de Compostela.

Villares, R. (2003). La ciudad de los dos Apóstoles (1875-1936). En E. Portela Silva (coord.), Historia de la ciudad de Santiago de Compostela (pp. 477-558). Santiago de Compostela: Servicións de Publicacións de la Universidad de Santiago de Compostela.

Xunta de Galicia, M. do P. G. (eds.) (2009). Olladas dunha época. Fotografias do Blanco-Cicerón. Pontevedra: Xunta de Galicia y Museo do Pobo Galego.

Fecha de recepción: 29/01/2020

Fecha de aceptación: 06/04/2020 